\title{
Production of Functional Carbon by Iron-Catalyzed Carbonization of Biomass - Effect of Washing with Acid Followed by Atmospheric Oxidation on the Electroconductivity of Crystallized Mesoporous Wood Carbon -
}

\author{
Tsutomu Suzuki ${ }^{1}{ }^{* *}$, Tatsuya Shinomoto ${ }^{1}$, Hiroshi Matsuzaki ${ }^{1}$, Kyoko Suzuki ${ }^{1}$, \\ Noriyasu Okazaki ${ }^{1}$, Yukie Saito ${ }^{2}$, Hidetoshi Kita ${ }^{3}$, and Hisashi Tamai ${ }^{4}$ \\ ${ }^{1}$ Department of Biotechnology and Environmental Chemistry, Kitami Institute of Technology, \\ 165 Koen-cho, Kitami, Hokkaido 090-8507, Japan \\ ${ }^{2}$ Graduate School of Agricultural and Life Science, The University of Tokyo, \\ 1-1-1 Yayoi, Bunkyo Ward, Tokyo 113-8657, Japan \\ ${ }^{3}$ Graduate School of Science and Engineering, Yamaguchi University, \\ 2-16-1 Tokiwadai, Ube, Yamaguchi 755-8611, Japan \\ ${ }^{4}$ Graduate School of Engineering, Hiroshima University, \\ 1-4-1 Kagamiyama, Higashihiroshima, Hiroshima 739-8527, Japan \\ *Fax: 81-157-24-7719, e-mail: suzuki@serv.chem.kitami-it.ac.jp
}

\begin{abstract}
Crystallized mesoporous wood carbon prepared at $850^{\circ} \mathrm{C}$ via two steps iron-catalyzed carbonization was washed with acid and then oxidized in air at $420^{\circ} \mathrm{C}$ for removal of iron and elimination of amorphous portion, respectively. The resulting carbon composed of graphitic nano-shell chains (GNSC) was equal or superior to highly electroconductive carbon, Ketjen black, in the ability as filler. The unique morphology of GNSC was featured by adequate crystallization and well developed mesoporosity in terms of the conductivity to achieve high dispersion in the matrix. Measurement of the quasi-inherent conductivity made in this connection disclosed the higher aptitude for this unusual woodderived carbon than for Ketjen black.

Key words: crystallized mesoporous wood carbon, iron-catalyzed carbonization, electroconductivity, graphitic nano-shell chain
\end{abstract}

\section{INTRODUCTION}

As wood is the most abundant biomass of good quality, this renewable and carbon-neutral resource is desirable to be converted into not only fluid fuels but also chemicals and materials as substitutes for petroleum. In response to this concept consistent with the current trend of biorefinery [1], we are now developing the two steps process of (1) carbonization of wood loaded with iron precursor at $500^{\circ} \mathrm{C}$ and (2) subsequent thermochemical treatment of obtained $500^{\circ} \mathrm{C}$-char at higher temperatures. The objective of step (1) is to make fine iron particles homogeneously distribute in the wood char, as shown in Fig. 1, together with the production of light oil as liquid fuel, whereas step (2) aims at anew affording useful functions to the resulting char. It should be noted in this connection that such nano-size iron particulates formed in the step (1) exhibit high catalytic activity at near $900^{\circ} \mathrm{C}$ which is the maximum operation temperature of step (2). This explains why the $500^{\circ} \mathrm{C}$-char can be efficiently converted into crystallized mesoporous carbon (abbreviated as CMC) having the dual function of electroconductivity and liquid phase adsorption capacity of macromolecules by $850^{\circ} \mathrm{C}$-carbonization as step (2) [2]. The concurrent evolution of hydrogen in a relatively large amount during the step (2) will raise the industrial attractiveness of the two steps iron-catalyzed carbonization process. However, all of related technologies are not satisfactorily completed. One of our present efforts is directed to exploring post-treatments for adequately

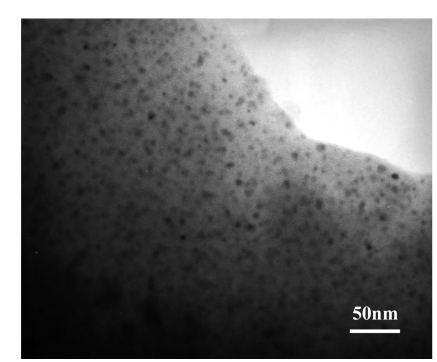

Fig. 1 TEM image of iron-loaded wood char: small black dots indicate iron particles.

enhancing the conductivity and/or the adsorption capacity of the iron-loaded CMC (Fe-CMC), and concerning the former function, washing with acid to remove iron for the recovery and reuse was found to be effective [3]. The other effort is addressing elucidation of the catalytic mechanism of the metal on the simultaneity of the crystallization of carbon and the development of mesoporosity.

Recently acid-treated $\mathrm{Fe}-\mathrm{CMC}$ (AFe-CMC) was carefully oxidized in air to selectively eliminate the amorphous portion, thereby appearing the unique morphology of graphitic nano-shell chains (GNSC) displayed in Fig. 2 [4]. A similar morphology was earlier observed as the substantial crystal structure of nickelloaded CMC prepared at $900^{\circ} \mathrm{C}$-carbonization [5]. There 


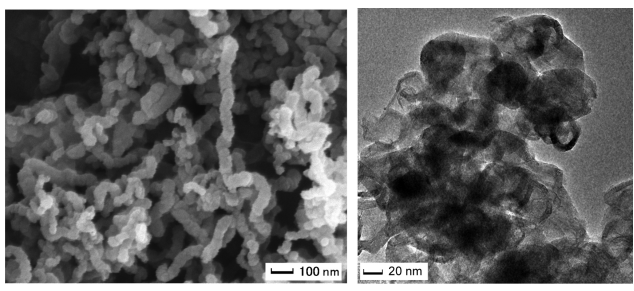

Fig. 2 SEM and TEM images of graphitic nano-shell chains formed in iron-loaded wood carbon.

is thus no doubt that the formation of GNSC through migration and agglomeration of iron particulates with several nano-meter in the cell wall matrix will hold the key to the relevant catalysis. A practical interest, on the other hand, is how the oxidized AFe-CMC (OAFe-CMC) will differ from $\mathrm{AFe}-\mathrm{CMC}$ in the function. To be concrete, washing with acid followed by controlled atmospheric oxidation is likely to be a more favorable post-treatment than the former alone for the conductivity. The present work focuses on this problem from a viewpoint of application of OAFe-CMC as the filler. In relation with the application, the role and/or effect of atmospheric oxidation are also discussed.

\section{THEORY}

For electroconductive carbon black (CB), adopted for comparison as described below, development of the relevant function is generally understood by the so-called percolation theory. According to this theory, the following characteristics are required for high ability [6, 7]: (i) small primary particle, (ii) large specific surface area, (iii) few impurity on the surface, (iv) well developed 'structure' (high fusion among primary particles), (v) high graphitization, and (vi) few functional groups capturing $\pi$ electrons. Factors (i), (ii), and (iii) facilitate the generation of conductivity circuit by increasing the particle density per unit weight, while factors (iv), (v), and (vi) contribute to promotion of the original conductivity circuit. The actual conductivity of $\mathrm{CB}$ itself is thus determined as a result of complicated interaction of many factors, but this value is not the theoretical 'inherent' value. The circumstances common to other conductive carbons like graphite, vapor-grown carbon fibers, etc. signify difficulty in specifying what factors are predominant. As a matter of fact, no exact definition or evaluation of the 'inherent' conductivity for all kinds of carbon materials is established, although the standard like JIS for CB [8] is set for the purpose and use. This is quite inconvenient for assessing the basic aptitude of such new carbons as wood-derived CMC and GNSC, because the applicability of the standard for $\mathrm{CB}$ is limited on account of their different fine structures and physicochemical properties. Needed are some proper devices or methods to systematically estimate the "quasiinherent' value for all carbons. For practical CB-derived conductive materials to be made by molding after kneading or mixing with a binder as the matrix, additional two factors must be taken in consideration: (vii) high dispersion of $\mathrm{CB}$ particles in the matrix and (viii) direct contact or mutual contiguity among $\mathrm{CB}$ particles in the composite structure [9]. As the final performance is dominated by factors (vii) and (viii), choice of the matrix is likewise critical for wood-derived carbon.

\section{EXPERIMENTAL}

3.1 Wood carbon

Powdered Japanese larch with diameter of $0.50-1.40$ mm was loaded with $\mathrm{Fe}\left(\mathrm{NO}_{3}\right)_{3} \cdot 9 \mathrm{H}_{2} \mathrm{O}$ by wet impregnation. The amount of iron was adjusted to 3 wt $\%$ as metal. After drying, the iron-loaded wood was carbonized in a downdraft tube reactor at $500^{\circ} \mathrm{C}$ for $1 \mathrm{~h}$ in a flow of nitrogen, and subsequently the $500^{\circ} \mathrm{C}$-char was subjected to $850^{\circ} \mathrm{C}$-carbonization for $1 \mathrm{~h}$ to prepare the starting material, Fe-CMC. The same two steps carbonization was done for raw wood powder to obtain the $850^{\circ} \mathrm{C}$-char as control. This char was denoted by NoWC. The yield on a dry ash-free, the metal-free basis of wood was about $24 \%$ and $23 \%$ for Fe-CMC and No$\mathrm{WC}$, respectively.

3.2 Washing with acid and atmospheric oxidation

$\mathrm{Fe}-\mathrm{CMC}$ was soaked in $1 \mathrm{M} \mathrm{HNO}_{3}$ with stirring for 12 $\mathrm{h}$ at room temperature for removal of iron. After washing with distilled water and drying at $105^{\circ} \mathrm{C}$, the acid-treated sample was recovered as $\mathrm{AFe}-\mathrm{CMC}$. Its recovery relative to $\mathrm{Fe}-\mathrm{CMC}$ was about $97 \%$ when corrected the content of iron. Atmospherically oxidized carbon, OAFe-CMC, was prepared from $\mathrm{AFe}-\mathrm{CMC}$ by heating it in a muffle furnace at $420^{\circ} \mathrm{C}$. The treatment period was controlled so as to reach the weight loss of $30 \%$, corresponding to the proportion of amorphous carbon in AFe-CMC. This proportion was in advance determined from the weight decrease by high frequency induction heating in vacuum up to $1800^{\circ} \mathrm{C}$ (SK Medical Electronics MU-1700 D).

3.3 Properties of carbon

For all wood carbon samples, both X-ray diffraction with $\mathrm{Cu}-\mathrm{K} \alpha$ radiation (XRD, Rigaku RINT 1200) and Laser Raman spectroscopic analysis with Ar beam of $514.5 \mathrm{~nm}$ (LRS, JUSCO NR-1800) were conducted to check the crystallinity of carbon. In XRD, intensity of the peak at about $26^{\circ}$ assigned to the crystallized form called T component was used for this purpose. In LRS, R value corresponding to the ratio of $\mathrm{D}$ band at $1360 \mathrm{~cm}^{-1}$ to $\mathrm{G}$ band at $1580 \mathrm{~cm}^{-1}$ was calculated as the index. Pore structure was examined from adsorption and desorption isotherms of nitrogen taken at $-196^{\circ} \mathrm{C}$ (ThermoQuest Sorptomatic 1990). These three measurements were likewise made with two commercial CB for comparison: Denka black (DB, a sort of acetylene black) and Ketjen black EC (KB) with medium and excellent conductivities, respectively. For the content of iron, atomic absorption spectrometric analysis (HITACHI Z-8200) was applied for the incombustible residue dissolved with aqua regia to quantify the metal.

\subsection{Preparation of a film specimen}

Each carbon sample was ground together with polytetrafluoroetylene in a mortar at the weight ratio of 9:1. The resulting small lumps were next pressed at 200 $\mathrm{kgf} \cdot \mathrm{cm}^{-2}$ for $20 \mathrm{~min}$ to extend into a sheet with thickness of about $250 \mu \mathrm{m}$. From the sheet, circle films with diameter of $10 \mathrm{~mm}$ were punched for the purpose of measuring the 'quasi-inherent' conductivity described in 3.6. This filming method is basically the same as that adopted to prepare carbon electrode for electric double layer capacitor. 
3.5 Pulverization with binder and molding into a disk specimen

All of carbon samples were individually pulverized in a planetary ball mill (FRITSH P-7) after mixing with cellulose acetate (CA), carboxymethyl cellulose (CC), or cornstarch (CS) as binder at different weight ratios. These biopolymer binders were powdered to $<0.15 \mathrm{~mm}$ in diameter prior to mixing. In the pulverization, the mixture was rotated at $630 \mathrm{rpm}$ and $2 \times 10 \mathrm{~min}$ together with agate balls of $15 \mathrm{~mm} \Phi$. The pulverized mixture was then molded in a stainless steel vessel by hot press at $140^{\circ} \mathrm{C}$ and $200 \mathrm{kgf} \cdot \mathrm{cm}^{-2}$ for $20 \mathrm{~min}$. The resulting disk was $30 \mathrm{~mm}$ and $1.6 \pm 0.1 \mathrm{~mm}$ in diameter and thickness, respectively.

3.6 Measurement of electroconductivity

The above film specimens were subjected to both measurements of surface resistivity and volume resistivity with a four-point probe [Dia Instruments, Loresta-GP]. For disk specimens, only volume resistivity was measured from a practical point.

\section{RESULTS AND DISCUSSION}

4.1 Crystallinity of carbon and pore structure

Figure 3 illustrates XRD profiles of all carbon samples. As already ascertained [4], both No-WC and KB were amorphous and DB was slightly crystallized. For three CMCs, their relevant peak intensity cannot correspond to the actual crystallinity correctly, because coexisted iron will absorb the radiation strongly [4]. To the contrary, $\mathrm{R}$ value obtained by LRS was helpful in confirming that the crystallinity was $\mathrm{Fe}-\mathrm{CMC}<\mathrm{AFe}-\mathrm{CMC}<\mathrm{OAFe}-\mathrm{CMC}$ with a small difference, as given in Table 1 . Although this index was substantially independent of the content of iron, its lower value for $\mathrm{DB}$ than for $\mathrm{AFe}-\mathrm{CMC}$ came into conflict with the information from XRD, suggesting the invalid application to quite different carbon materials in the basic crystal form. Consequently, the whole crystallinity determined by XRD combined with LRS was No-WC $\fallingdotseq \mathrm{KB}<\mathrm{DB}<\mathrm{Fe}-\mathrm{CMC}<\mathrm{AFe}-\mathrm{CMC}<$ OAFe-CMC. In Fig. 4, curves of adsorption (lower) and desorption (upper) are depicted for each carbon. The pattern up to about 0.5 of $\mathrm{P} / \mathrm{P}_{0}$ indicated $\mathrm{OAFe}-\mathrm{CMC}<$ $\mathrm{DB}, \mathrm{AFe}-\mathrm{CMC}, \mathrm{Fe}-\mathrm{CMC}<\mathrm{No}-\mathrm{WC} \ll \mathrm{KB}$ in $\mathrm{BET}$ surface area. Mesoporosity judged from the hysteresis at $\mathrm{P} / \mathrm{P}_{0}$ of $0.5-0.9$ was $\mathrm{DB}, \mathrm{No}-\mathrm{WC} \ll \mathrm{Fe}-\mathrm{CMC}, \mathrm{AFe}-\mathrm{CMC}$ $<\mathrm{OAFe}-\mathrm{CMC}<\mathrm{KB}$. It was also found that OAFe-CMC resembled $\mathrm{KB}$ in the distribution, or constitution of

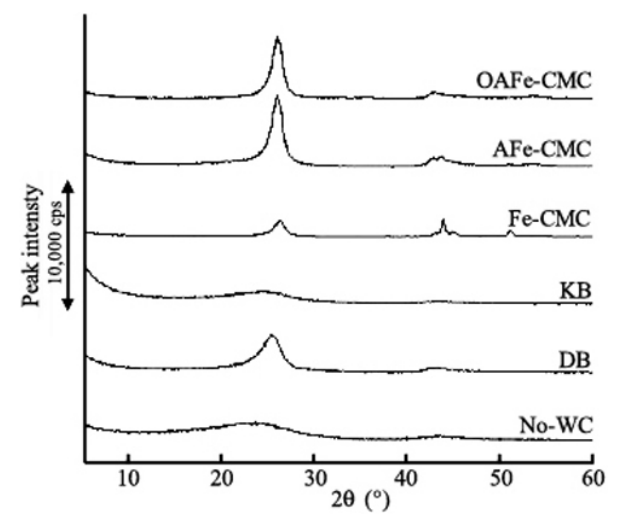

Fig. 3 XRD profiles for all carbon samples.
Table $1 \mathrm{R}$ value and iron content for each carbon

\begin{tabular}{c|cc}
\hline Sample & R value $(-)^{\mathrm{a})}$ & Iron $(\mathrm{wt} \%)$ \\
\hline No-WC & 1.81 & $<0.1$ \\
Fe-CMC & 1.52 & 8.8 \\
AFe-CMC & 1.50 & 1.0 \\
OAFe-CMC & 1.48 & 1.5 \\
DB & 1.38 & $<0.1$ \\
KB & 1.56 & $<0.1$ \\
\hline
\end{tabular}

a) See the text.

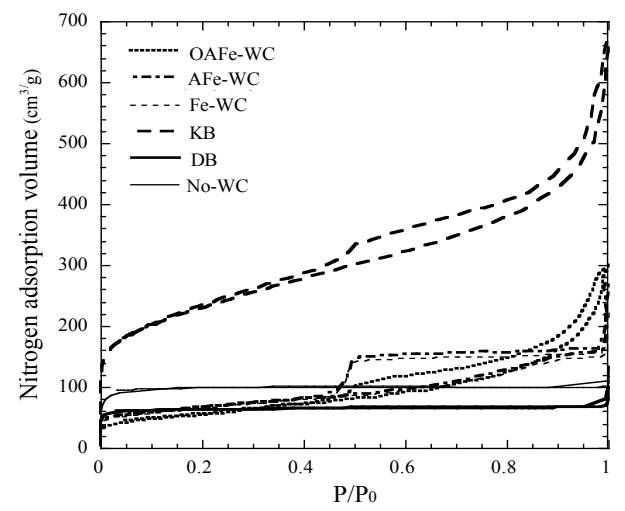

Fig. 4 Adsorption and desorption curves of nitrogen for each carbon.

mesopore. These situations made clear little change of pore structure by the removal of iron and a marked enrichment with mesopore for OAFe-CMC.

4.2 Quasi-inherent electroconductivity

Figure 5 compares surface and volume resistivities of film specimens for all carbon samples. This result revealed that the 'quasi-inherent' conductivity was No$\mathrm{WC} \ll \mathrm{DB}<\mathrm{KB} \ll \mathrm{Fe}-\mathrm{CMC} \fallingdotseq \mathrm{AFe}-\mathrm{CMC}<\mathrm{OAFe}-\mathrm{CMC}$. Much better performance for three CMCs than for two commercial carbons seemed difficult to be accounted by only higher crystallinity of carbon relating to factor (v). Although the difference among three CMCs and between $\mathrm{DB}$ and KB implied the importance of not factor (ii) but the revised factor (ii') replacing 'specific surface area' by 'mesopore surface area', OAFe-CMC was much inferior to $\mathrm{KB}$ in the exact parameter. The most plausible interpretation at the present stage was thus so effective formation of GNSC as to make factors (vi) and/or (i) favorable. In brief, the excellent conductivity of OAFe$\mathrm{CMC}$ can be accepted as a result of the synergic

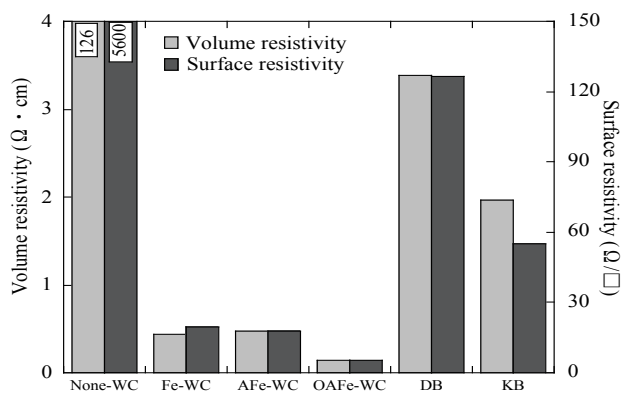

Fig. 5 Quasi-inherent conductivity for each carbon. 
improvement of factors (i), (ii'), (iv), and (v). No great difference for $\mathrm{Fe}-\mathrm{CMC}$ and $\mathrm{AFe}-\mathrm{CMC}$ signified little disadvantage of coexisted iron, thereby explaining that factors (iii) and (vi) were unimportant.

4.3 Influence of binder

Table 2 exemplifies the influence of $\mathrm{CA}, \mathrm{CC}$, and CS on volume resistivity of disk specimens for three $\mathrm{CMCs}$ at the weight ratio of $1: 1$. The conductivity of $\mathrm{Fe}-\mathrm{CMC}<$ $\mathrm{AFe}-\mathrm{CMC}<\mathrm{OAFe}-\mathrm{CMC}$ regardless of the matrix would reflect the unfavorable coexistence of iron and the favorable development of mesoporosity, although the order was not always inconsistent with Fig. 5 The suitability as the matrix resulted in $\mathrm{CS}<\mathrm{CC}<\mathrm{CA}$, because the conductivity for any $\mathrm{CMC}$ enhanced in this order at different weight ratios. Accordingly, CA surpassed $\mathrm{CS}$ and $\mathrm{CC}$ in the matrix effect having relation with factors (vii) and (viii)

Table 2 Influence of binder on the conductivity of three $\mathrm{CMCs}$ at the ratio of $1: 1$ in weight.

\begin{tabular}{c|cc}
\hline Sample & Binder $^{\mathrm{a})}$ & $\begin{array}{c}\text { Volume resistivity } \\
(\Omega \cdot \mathrm{cm})\end{array}$ \\
\hline Fe-CMC & CA & 0.24 \\
& CC & 0.34 \\
AFe-CMC & CS & 0.84 \\
& CA & 0.11 \\
& CC & 0.24 \\
OAFe-CMC & CS & 0.28 \\
& CA & 0.07 \\
& CC & 0.19 \\
& CS & 0.25 \\
\hline
\end{tabular}

a) See the text ${ }^{\text {a) }}$

4.4 Relation of the conductivity with the proportion in weight

Figure 6 represents the relation of volume resistivity with the proportion in weight up to $40 \%$ for disk specimens blended with CA. At less than 20 wt $\%$, the conductivity was $\mathrm{No}-\mathrm{WC} \ll \mathrm{Fe}-\mathrm{CMC}<\mathrm{AFe}-\mathrm{CMC}<\mathrm{DB}$ $<\mathrm{OAFe}-\mathrm{CMC} \leqq \mathrm{KB}$. The fact that OAFe-CMC was superior and comparable to $\mathrm{DB}$ and $\mathrm{KB}$, respectively, gave evidence of the high practical ability of GNSC as filler. Although CA was not necessarily a proper matrix for $\mathrm{DB}$ and $\mathrm{KB}$, their superiority over AFe-CMC opposite to the above 'quasi-inherent' conductivity resulted from adequately high dispersion of these $\mathrm{CBs}$, thus recognizing the significance of factor (vii) again. Conversely speaking, poor dispersion in the matrix of $\mathrm{AFe}-\mathrm{CMC}$ made the high potentiality spoil. It became also certain that poorer dispersion of $\mathrm{Fe}-\mathrm{CMC}$ was ascribed to the high content of iron. However, at over 20 $\mathrm{wt} \%$ where factor (viii) would have priority over factor (vii), OAFe-CMC exceeded $\mathrm{KB}$ due to failed molding for the latter: this $\mathrm{CB}$ is too bulky, or incompressible to be molded in such a high proportion range. Although DB is not so bulky as $\mathrm{KB}$, the increment in the conductivity by increasing the proportion from 20 to $40 \mathrm{wt} \%$ was much smaller than those for AFe-CMC and Fe-CMC. The common feature of three CMCs that higher conductivity can be gained by higher weight ratio without losing the formability is a big advantage in the industrial application. Exploration of more proper binders than $\mathrm{CA}$ to improve the dispersion of OAFe$\mathrm{CMC}$ is a chief subject of our further study.

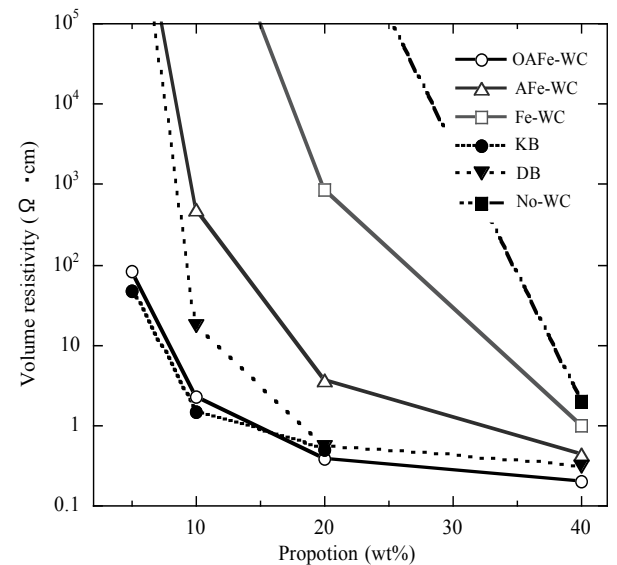

Fig. 6 The change of electroconductivity with the proportion in weight for each carbon.

\section{CONCLUSION}

The present work demonstrated that $\mathrm{OAFe}-\mathrm{CMC}$ with the morphology of GNSC was superior or comparable to commercial excellent conductive $\mathrm{KB}$ in the ability as filler. In parallel, the order of Fe-CMC $<$ AFe-CMC $<$ $\mathrm{OAFe}-\mathrm{CMC}$ in the relevant ability was elucidated. It was also deduced from the 'quasi-inherent' conductivity that the three CMCs had originally higher aptitude for the conductivity than KB. These findings and information were valuable enough to make the production of highly electroconductive carbon from wood by the two steps iron-catalyzed carbonization more attractive.

\section{ACKNOWGEDGMENT}

This work was supported by a Grant-in-Aid for Scientific Research from the Ministry of Education, Culture, Sports, Science and Technology, Japan (No.21380106).

[1] http://www.nrel.gov/biomass/biorefinery.html; http:// www.biorefinery-euroview.eu/biorefinery/public/index. html; http://www.alpenabiorefinery.com/index.html, etc.

[2] T. Suzuki, J. Jpn. Inst. Energy, 89, 373-376 (2010) (in Japanese).

[3] T. Suzuki, H. Matsuzaki, K. Suzuki, Y. Saito, S. Yasui, N. Ozaki, T. Yamada, Chem. Lett., 37, 798-9 (2008).

[4] K. Suzuki, T. Suzuki, Y. Saito, H. Kita, K. Sato, T. Konno, Abstracts of the 37th annual meeting of the Carbon Society of Japan, pp. 58-59 (2010).

[5] K. Suzuki, T. Suzuki, Y. Saito, H. Kita, K. Sato, T. Konno, Tanso, 239, 169-71 (2009) (in Japanese).6

[6] TRC R\&D Library, "Carbon materials in the field of electronics", p.112 (2003) (in Japanese).

[7] S. Maeno, "The latest complete technology of carbon black", Technical Information Institute Co. Ltd., Tokyo, pp. 234-235 (2005) (in Japanese).

[8] http://www.tansokyoukai.biz-web.jp/jis-jcas.pdf

[9] Y. Fukuyama, "The latest complete technology of carbon black", Technical Information Institute Co. Ltd., Tokyo, p. 246 (2005) (in Japanese).

(Received February 17, 2011; Accepted June 15, 2011) 